\section{Polypharmacy in the terminally ill}

To THE EDITOR: A significant number of patients who receive palliative care are over 65 years of age. In addition to their terminal illness, they often have other medical comorbidities. Long-term medical management of these conditions can evolve into a large regimen of medications. Patients may continue to take these medications as they enter the terminal phase of their illness, but it is often unnecessary and to their detriment. $^{1}$

We performed a retrospective chart review between 8 July and 6 October 2010 to determine the prevalence of polypharmacy in 50 consecutive patients aged over 65 years who were admitted to the Palliative Care Unit (PCU) at Redcliffe Hospital and who died during that admission. Because patients receiving palliative care are invariably prescribed several medications for symptom control (analgesics, laxatives and antiemetics), we adopted a liberal total as the cut-off and deemed that nine or more medications constituted polypharmacy for this cohort.

The mean age of patients was 76.9 years and 17 were women. Polypharmacy was prevalent (21 patients). The median number of medications per patient on admission was seven, with men prescribed a median of seven, and women a median of eight. Patients aged 66-74 years were on a median of 10 medications compared with seven for patients aged 75 years and over. The total number of medications prescribed to the cohort of 50 patients at the time of admission was 427 . By 72 hours after admission, 285 of these (67\%) had been ceased. The median time from the patient's admission to their death was 7 days.

The results of our study suggest a need for vigilance in identifying polypharmacy in the terminally ill. Effective management of polypharmacy involves discontinuing medications deemed no longer necessary, if it is safe to do so. This can be achieved by regular review of a palliative care patient's medications by the physicians involved in their care - for example, the primary care physician, oncologist and palliative care physician - as the patient approaches the terminal phase of their illness. In our experience, regular review is more likely to be done if one physician is identified as having responsibility for this.

Ross P Cruikshank Registrar in Medical Oncology and Palliative Care ${ }^{\mathrm{T}}$

Bruce Stafford Director of Palliative Care ${ }^{1}$

Lee Jones Statistician²

1 Redcliffe Hospital, Redcliffe, QLD.

2 Queensland Institute of Medical Research, Brisbane QLD.

\section{rosscruikshank@yahoo.com}

Competing interests: No relevant disclosures.

doi: 10.5694/mjal2.11660

1 Basger BJ, Chen TF, Moles RJ. Inappropriate medication use and prescribing indicators in elderly Australians: development of a prescribing indicators tool. Drugs Aging 2008; 25: 777-793.

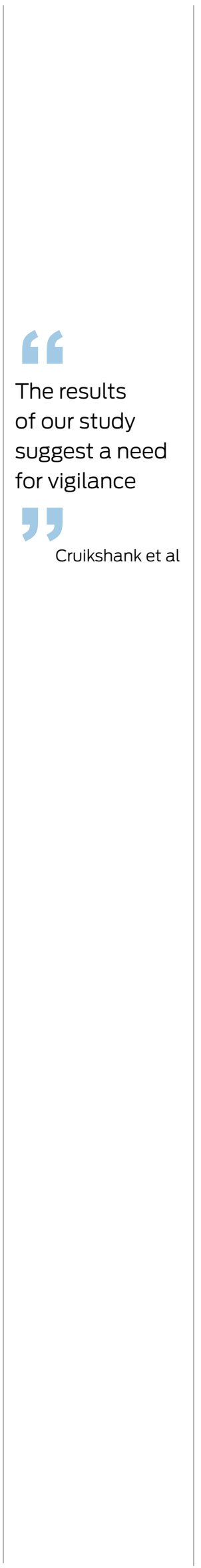

